\title{
Histopathological Changes in Breast Cancers Following Neoadjuvant Chemotherapy: Implications for Assessment of Therapy-Induced Cytological and Stromal Changes for Better Clinical Outcome and Effective Patient care
}

\author{
Shazima Sheereen ${ }^{1}$ Flora D. Lobo ${ }^{1}$ Barun Kuma \\ Waseemoddin Patel ${ }^{5}$ Abhishek Singh Nayyar ${ }^{6}$ \\ ${ }^{1}$ Department of Pathology, Kasturba Medical College, Manipal Academy \\ of Higher Education, Mangalore, Karnataka, India \\ ${ }^{2}$ Department of Oral and Maxillofacial Surgery, Bharati Vidyapeeth \\ (Deemed to be University) Dental College and Hospital, Sangli, \\ Maharashtra, India \\ ${ }^{3}$ Department of Oral Radiology, College of Dentistry, University of Ha'il, \\ Ha'il, Kingdom of Saudi Arabia \\ ${ }^{4}$ Department of Oral and Maxillofacial Surgery, Malla Reddy Dental \\ College for Women, Hyderabad, Telangana, India \\ ${ }^{5}$ Department of Pediatrics and Neonatology, McMaster University, \\ Hamilton, Ontario, Canada \\ ${ }^{6}$ Department of Oral Medicine and Radiology, Saraswati- \\ Dhanwantari Dental College and Hospital and Postgraduate \\ Research Institute, Parbhani, Maharashtra, India
}

Address for correspondence Shazima Sheereen, Department of Pathology, Kasturba Medical College, Manipal Academy of Higher Education, Mangalore, Karnataka, India (e-mail: dr.shazimasheereen@gmail.com).

\begin{abstract}
Keywords

- therapy-induced histopathological changes

- breast cancers

- neoadjuvant chemotherapy

- breast cancer diagnosis and treatment

The histopathological interpretation of a tumor still remains the gold standard for diagnosis and deciding the type of therapy. Furthermore, in the context of chemo-radio-therapies often leading to prominent cytological and stromal changes in the tumor, histopathological interpretation during treatment, thus, becomes all the more important in correctly diagnosing and grading the tumor for an effective and planned regimen of the therapy increasing prognosis and chances of survival of the affected patients. The aim of the present study was to evaluate such cytological and stromal changes rendered by the therapy during treatment in breast cancer cases. The present study was conducted over a period of 4 years from January 2014 to June 2017 at Kasturba Medical College, Mangalore, Karnataka, India wherein the clinical and histopathological details were collected for a total of 39 breast carcinoma cases during and post-therapy. Statistical analysis was done using IBM SPSS statistics 17 (Chicago, USA). Various morphological features were analyzed for their frequency and were compared with the final diagnosis using Chi-square value $\left(\chi^{2}\right)$, paired t-test and Fischer's test. $p$-value $<0.05$ was considered to be statistically significant. The results of the present study revealed that 32 of the 39 breast carcinoma cases changed their grades during the course of therapy ( $p$-value $<0.05$ ). The fundamental manifestation of the effect of therapy was an obvious decrease in tumor cellularity.
\end{abstract}

DOI https://doi.org/ $10.1055 / \mathrm{s}-0038-1676909$ ISSN 2454-6798.
(C)2018 Spring Hope Cancer Foundation \& Young Oncologist Group of Asia
License terms

(이 (1) $\Theta \circledast$ 
Intracellular changes commonly noted after chemotherapy included nuclear enlargement, hyperchromasia and an increased $\mathrm{N}$ : C ratio which were found in upto $85 \%$ of the cases followed by presence of prominent nucleoli and karyorrhexis/karyolysis. The predominant stromal changes seen post-therapy included necrosis (74.4\%), fibrosis (64.1\%), desmoplasia (59\%) and degenerative changes (33.3\%). Breast cancer therapy causes morphological alterations in the cancerous as well as the surrounding healthy tissue. The histopathological interpretation in such cases, thus, requires a thorough knowledge of the cytological and stromal changes rendered by the therapy.

\section{Introduction}

Breast cancer is the most common cancer diagnosed in women and is the second leading cause of death in women globally. ${ }^{1}$ Even though most cases occur over the age of 50 years, it is not uncommon in younger women. ${ }^{1,2}$ Breast cancer is not a single disease and comprises several entities ranging from ductal carcinoma in situ (DCIS) to a widespread metastatic disease. ${ }^{3}$ Clinical presentation and investigations such as mammography and fine-needle aspiration cytology (FNAC) often help in the diagnosis of breast cancers. However, histopathologic examination remains the better predictor for an accurate diagnosis of breast cancers. In addition, immunohistochemical markers aid in classification and are often used to guide the therapy of the disease process. Therapy and prognosis depend on the various clinical and histopathologic factors, including tumor size, type of tumor, hormonal receptor status, the type of therapy provided, and, most significantly, at what stage the cancer is diagnosed. Because of extensive advances made in the field of oncology, various modes of therapy of breast cancers are available at present including surgery, radiotherapy, chemotherapy, and, more specifically, targeted hormonal and tumor receptors targeted therapy. Surgery that may include mastectomy or, breast-conserving surgery (lumpectomy), still remains the prime modality of therapy for most of the operable breast cancers. Hormonal therapy can be a significant addition along with surgery or, chemotherapy, especially, in patients who are estrogen receptor (ER) and/or, progesterone receptor (PR) positive. Hormonal receptor status can, thus, significantly alter the modality of therapy provided and may have better outcomes. In advanced disease, prior to surgery, radiation therapy is used to reduce the tumor size. In early breast cancers, to facilitate breast conservation, chemotherapy is more commonly used both as a neoadjuvant chemotherapy and/or postoperative chemotherapy. ${ }^{4}$ In the modern era, increased number of neoadjuvant therapy-induced surgical resection specimens of tumors are being received in histopathologic evaluation laboratories. The pathologist evaluates the residual tumor both in the breast and lymph nodes. Assessment of the therapy-induced morphologic changes seen at the cytologic and stromal levels, thus, requires a thorough knowledge of the possible cytologic and stromal changes rendered by the therapy. The aim of this study was to evaluate such cytologic and stromal changes rendered by the therapy in breast cancer cases. This ensures the role of pathologists in the correct diagnosis as well as grading of the tumor with a correct histopathologic interpretation for an effective and planned regimen of the therapy increasing prognosis and chances of survival of the affected patients rendering better clinical outcome and an effective patient care.

\section{Materials and Methods}

This study was a combined retrospective and prospective study conducted over a period of 4 years from January 2014 to June 2017 at Kasturba Medical College, Mangalore, Karnataka, India. Clinical and histopathologic details were collected from a total of 39 cases of breast carcinoma before and post-therapy, and the changes induced by the therapy were correlated. All the patients who had been diagnosed with stages II and III (T1-T4, N0-2, M0) carcinoma breast were included in the study while patients already treated with chemo- or hormonal therapy and patients who only underwent surgery without chemo- or hormonal therapy and wherein biopsy samples were found to be inadequate were excluded. The required clinical data of the patients, their lymph node status, and investigation reports (mammography, FNAC, biopsy, ER/PR status, Her2/neu status) were collected before the start of the planned therapy. The initial biopsies were subjected to routine formalin fixation and paraffin processing with microscopic analysis on hematoxylin and eosin-stained sections supplemented by special stains including immunohistochemistry to decide the histologic types and grades of the tumor and hormonal status. Subsequently, when the patients received two to six cycles of chemotherapy or, hormonal therapy, histopathologic (gross and microscopic) and immunohistochemical examination was performed and all the details pertaining to microscopic and clinical findings along with immunohistochemical findings were recorded and the changes induced by therapy were correlated.

\section{Statistical Analysis}

Statistical analysis was done using IBM SPSS statistics 17 (Chicago, Illinois, USA). The data were analyzed by proportions, tables, and graphs whereas various morphologic features were analyzed for their frequency and 
were compared with the final diagnosis using chi-square value $\left(\chi^{2}\right)$, paired $t$-test, and Fischer's test. $p<0.05$ was considered to be statistically significant whereas $p<0.001$ was considered as highly significant.

\section{Results}

This study consisted of 39 breast carcinoma cases. The median age of patients was found to be 55 years. The most common chief complaint was a palpable swelling in the affected breast whereas mastalgia (38.5\%), discharge from nipple, and ulcer were the other common complaints. The right breast was more commonly involved (66.7\%), and upper and outer quadrant was the most common location of the tumor (74.4\%) followed by upper and inner quadrant (8\%). In $13 \%$ of the cases, more than one quadrant was involved. Most cases (69\%) in this study received four to six cycles of neoadjuvant chemotherapy whereas $31 \%$ of cases received more than six cycles of chemotherapy. AC regimen (doxorubicin, cyclophosphamide followed by paclitaxel) was the most common (26 cases, 64.1\%) chemotherapy regimen received. The second most common ( 8 cases, 20.5\%)

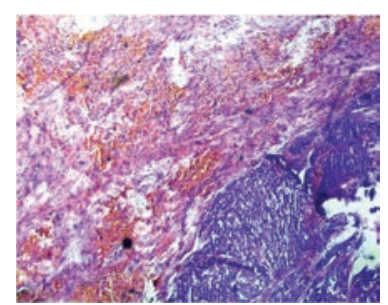

Fig. 1 IDC-NOC: Infiltrating ductal carcinoma-not otherwise specified: Post-therapy evaluation of tumor: Tumor cells (H\&E, 40x).

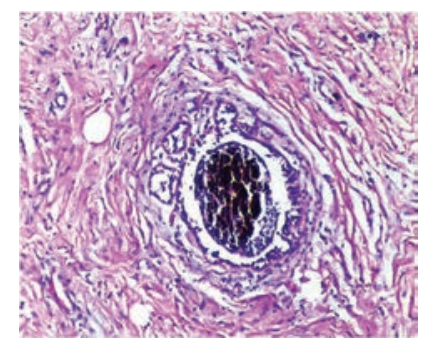

Fig. 3 IDC-NOC: Infiltrating ductal carcinoma-not otherwise specified: Post-therapy evaluation of tumor: Residual tumor cells showing areas of fibrosis and calcification (H\&E, 200x).

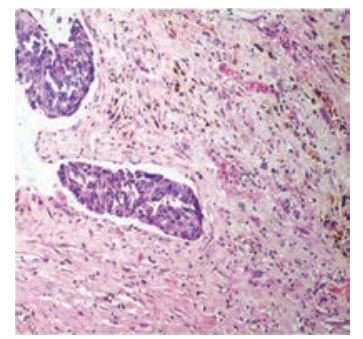

Fig. 5 IDC-NOC: Infiltrating ductal carcinoma-not otherwise specified: Post-therapy evaluation of tumor: Residual tumor cells showing hemosiderin laden macrophages along with areas of fibrosis (H\&E, 100x). regimen received was $\mathrm{CAF}$ (cyclophosphamide, doxorubicin, 5-fluorouracil) followed by CEF (cyclophosphamide, epirubicin, 5-fluorouracil) regimen (6 cases, 15.38\%). The mean size of the tumor before induction of chemotherapy was found to be $3.75 \mathrm{~cm}$ whereas after chemotherapy it was $1.75 \mathrm{~cm}(p<0.001)$. The tumor size decreased consistently after neoadjuvant chemotherapy. Stage II breast carcinoma was found to be the most predominant (61.5\%) stage with stage IIA accounting for up to $38.5 \%$ of the cases, and $23 \%$ of the cases were found to be in stage III. It was observed that younger women had an aggressive breast cancer with stage III as the predominant stage noted. IDCNOC (infiltrating ductal carcinoma-not otherwise specified) (-Figs. 1-15) was the most common histologic type both before $(94.87 \%)$ and after (76.92\%) therapy. One case of lobular carcinoma and one case of metaplastic carcinoma (-Figs. 16-18) were also found. Thirty-two of the 39 tumors changed grade to either a higher or lower grade $(p<0.05)$. On histopathologic examination, pathologic complete response (pCR) (-Figs. 19-21) was observed in $18 \%$ of the cases whereas $15 \%$ showed pathologic partial response (pPR) and $66.7 \%$ cases had a stable disease (SD) with no progression

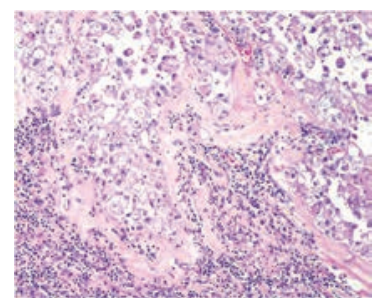

Fig. 2 IDC-NOC: Infiltrating ductal carcinoma-not otherwise specified: Post-therapy evaluation of tumor: Lymph nodal tissue showing residual tumor cells with areas of fibrosis and hyalinization (H\&E, 100x).

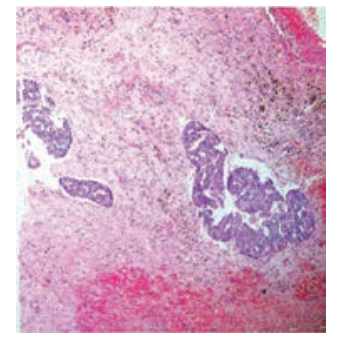

Fig. 4 IDC-NOC: Infiltrating ductal carcinoma-not otherwise specified: Post-therapy evaluation of tumor: Residual tumor cells showing hemosiderin laden macrophages along with areas of fibrosis (H\&E, 40x).

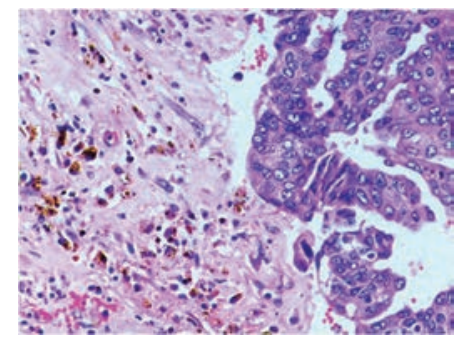

Fig. 6 IDC-NOC: Infiltrating ductal carcinoma-not otherwise specified: Post-therapy evaluation of tumor: Residual tumor cells showing hemosiderin laden macrophages along with areas of fibrosis (H\&E, 200x). 


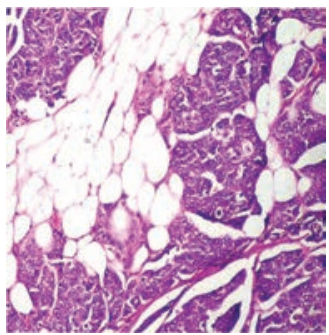

Fig. 7 IDC-NOC: Infiltrating ductal carcinoma-not otherwise specified: Post-therapy evaluation of tumor: Perinodal fat infiltration of tumor cells (H\&E, 100x).

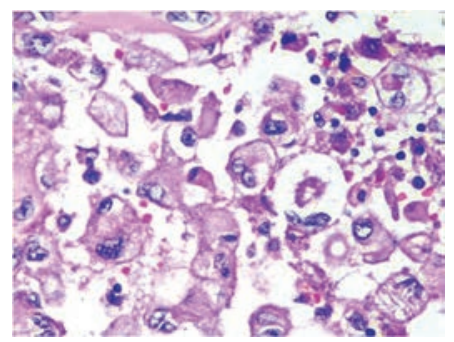

Fig. 9 IDC-NOC: Infiltrating ductal carcinoma-not otherwise specified: Post-therapy evaluation of tumor: Residual tumor cells in lymph nodal tissue (H\&E, 400x).

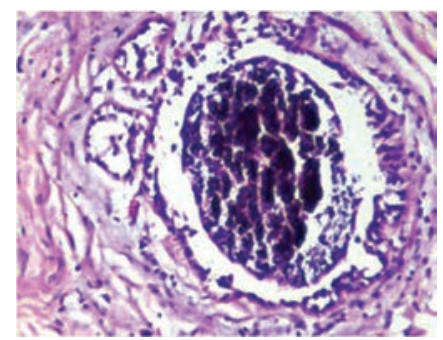

Fig. 11 IDC-NOC: Infiltrating ductal carcinoma-not otherwise specified: Post-therapy evaluation of tumor: Residual tumor cells showing areas of fibrosis and calcification (H\&E, 200x).

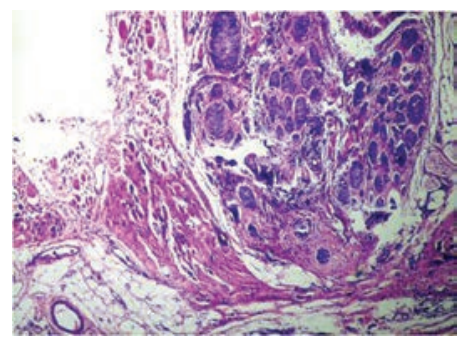

Fig. 13 IDC-NOC: Infiltrating ductal carcinoma-not otherwise specified: Post-therapy evaluation of tumor: Tumor in skeletal muscle (H\&E, 200x).

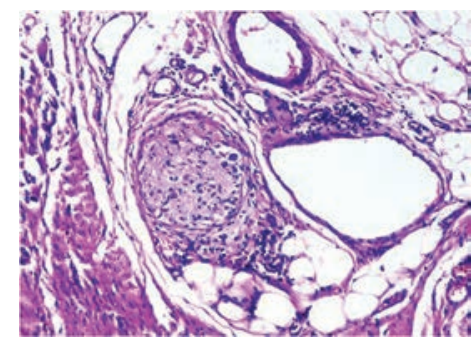

Fig. 15 IDC-NOC: Infiltrating ductal carcinoma-not otherwise specified: Post-therapy evaluation of tumor: Neural invasion (H\&E, 200x).

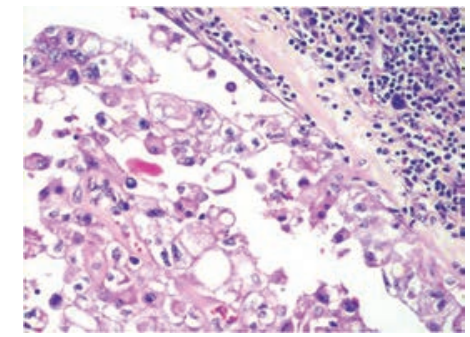

Fig. 8 IDC-NOC: Infiltrating ductal carcinoma-not otherwise specified: Post-therapy evaluation of tumor: Residual tumor cells in lymph nodal tissue (H\&E, 200x).

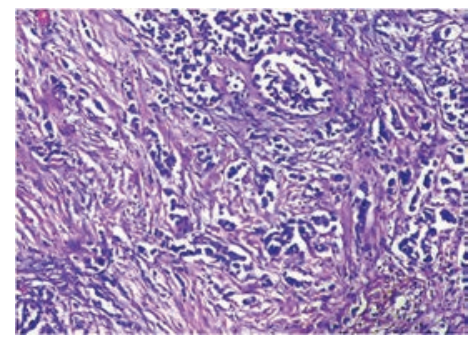

Fig. 10 IDC-NOC: Infiltrating ductal carcinoma-not otherwise specified: Post-therapy evaluation of tumor: Residual tumor cells showing areas of fibrosis and calcification (H\&E, 100x).

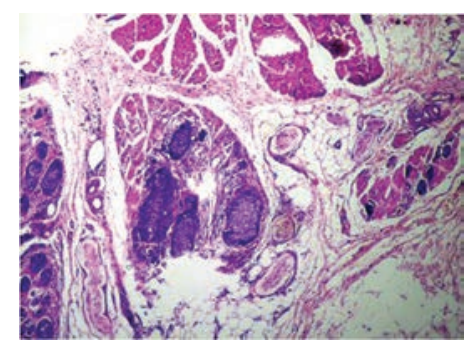

Fig. 12 IDC-NOC: Infiltrating ductal carcinoma-not otherwise specified: Post-therapy evaluation of tumor: Tumor in skeletal muscle (H\&E, 40x).

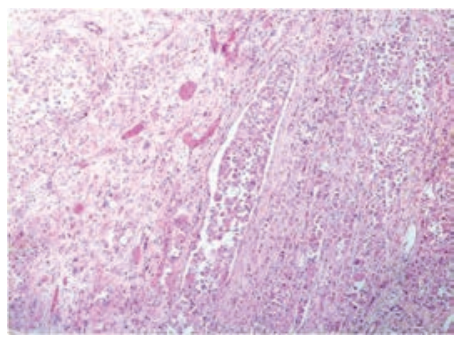

Fig. 14 IDC-NOC: Infiltrating ductal carcinoma-not otherwise specified: Post-therapy evaluation of tumor: Lymphovascular invasion (H\&E, 100x).

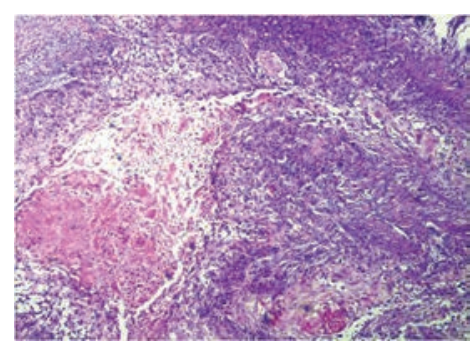

Fig. 16 Metaplastic carcinoma: Post-therapy evaluation of tumor: Metaplastic carcinoma with squamous differentiation (H\&E, 40x). 
of disease (-Table 1). The fundamental manifestation of the effect of therapy was an obvious decrease in tumor cellularity. Intracellular changes commonly noted after chemotherapy-included nuclear enlargement, hyperchromasia, and increased nuclear-to-cytoplasmic $(\mathrm{N}: \mathrm{C})$ ratio that were found in up to $85 \%$ of the cases, followed by presence of prominent nucleoli and karyorrhexis/karyolysis noted in 70 to $75 \%$ of the cases (-Table 2). The predominant stromal changes seen post-therapy were necrosis (74.4\%), fibrosis (64.1\%) (-Figs. 2-6, 10, 11, 19-21), desmoplasia (59\%), and degenerative changes (33.3\%). Other significant

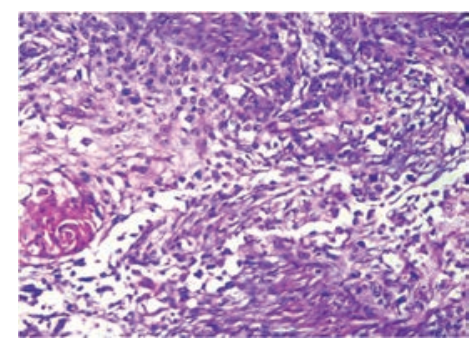

Fig. 17 Metaplastic carcinoma: Post-therapy evaluation of tumor: Metaplastic carcinoma with squamous differentiation (H\&E, 100x).

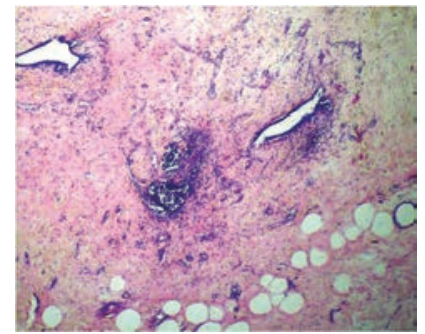

Fig. 19 pCR: pathologic complete response of breast cancer: Post-therapy evaluation of tumor: Fibrotic stroma (H\&E, 40x). stromal changes included mucinous changes, hyalinization of walls of blood vessels, ( - Fig. 2) neo-angiogenesis, calcific deposits ( - Figs. 3, 10, 11), loss of cellular architecture, and appearance of chronic inflammatory cell infiltration (-Figs. 20-21) and hemosiderin-laden macrophages (-Figs. 4-6), giant cells, and histiocytes (- Table 3).

\section{Discussion}

Breast cancer therapy causes morphologic alterations in the cancerous as well as the surrounding healthy tissue. However,

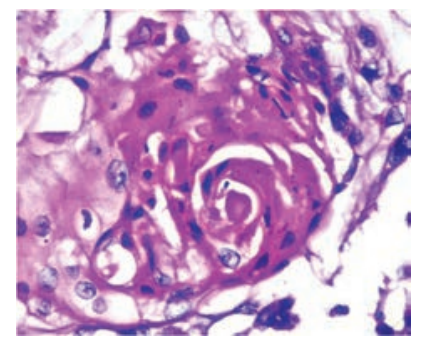

Fig. 18 Metaplastic carcinoma: Post-therapy evaluation of tumor: Squamous pearls (H\&E, 200x).
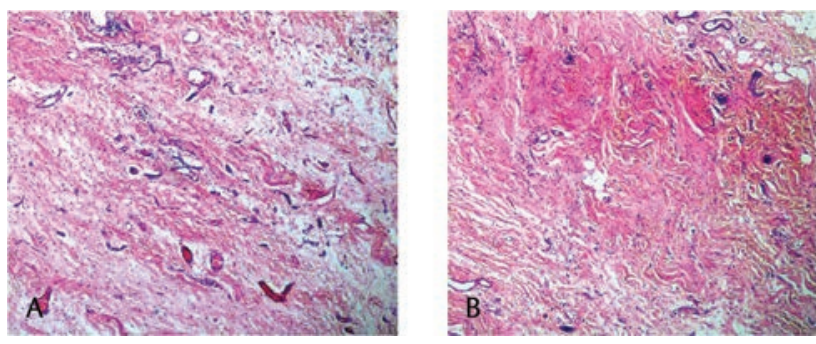

Fig. 20 (A,B) pCR: pathologic complete response of breast cancer: Post-therapy evaluation of tumor: Granulation tissue with areas of fibrosis, chronic inflammatory cell infiltration and hemorrhage.
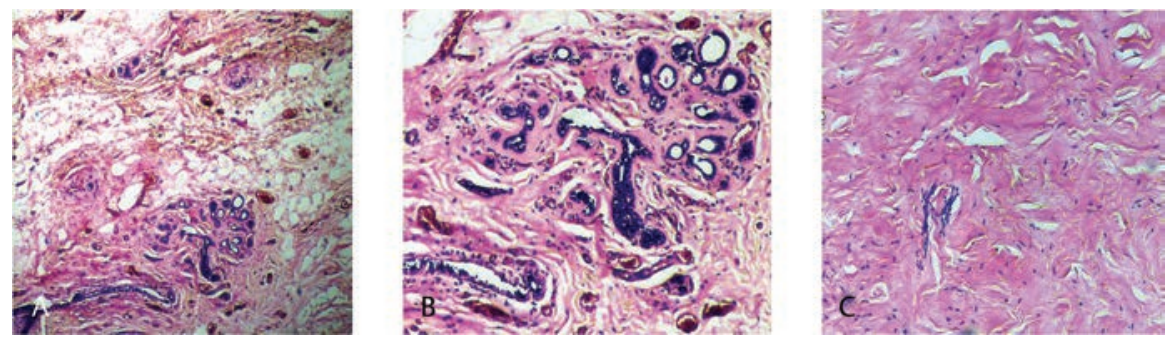

Fig. 21 (A-C) Inflammatory response with areas of fibrosis and absence of tumor cells.

Table 1 Carcinoma breast: comparison of effect of therapy

\begin{tabular}{|c|c|c|c|c|c|}
\hline $\begin{array}{l}\text { Effect of } \\
\text { therapy }\end{array}$ & $\begin{array}{l}\text { Obtained in study } \\
\text { conducted by van } \\
\text { der Wall et al }{ }^{18} \\
(n=62), n(\%)\end{array}$ & $\begin{array}{l}\text { Obtained in study } \\
\text { conducted by } \\
\text { Burcombe et al }{ }^{13} \\
(\boldsymbol{n}=99), \boldsymbol{n}(\%)\end{array}$ & $\begin{array}{l}\text { Obtained in } \\
\text { study conducted } \\
\text { by Moon et al }{ }^{25} \\
(n=82), n(\%)\end{array}$ & $\begin{array}{l}\text { Obtained in } \\
\text { study conducted } \\
\text { by Sethi et al }{ }^{6} \\
(n=40), n(\%)\end{array}$ & $\begin{array}{l}\text { Obtained in this } \\
\text { study }(n=39), n(\%)\end{array}$ \\
\hline $\mathrm{pCR}$ & $13 / 62(21)$ & $8 / 99(8)$ & $21(25)$ & $4 / 40(10)$ & $7 / 39(17.9)$ \\
\hline pPR & $42 / 62(68)$ & $30 / 99(30)$ & $49(59)$ & $12 / 40(30)$ & $6 / 39(15.4)$ \\
\hline pNR & $7 / 62(11)$ & $61 / 99(62)$ & $12(15)$ & $24 / 40(60)$ & $26 / 39(66.7)$ \\
\hline
\end{tabular}

Abbreviations: pCR, pathologic complete response; pNR, pathologic no response; pPR, pathologic partial response. 
Table 2 Carcinoma breast: correlation of nuclear changes after neoadjuvant therapy

\begin{tabular}{|l|l|}
\hline Nuclear response & Number of cases (\%) \\
\hline Nuclear enlargement & $35(89.7)$ \\
\hline Hyperchromasia & $34(87.2)$ \\
\hline $\begin{array}{l}\text { Increased nuclear-to-cytoplasmic } \\
\text { ratio }\end{array}$ & $33(84.6)$ \\
\hline Presence of prominent nucleoli & $30(76.9)$ \\
\hline Karyorrhexis/karyolysis & $28(71.8)$ \\
\hline Pyknosis & $23(59)$ \\
\hline Pleomorphic nuclei & $24(61.5)$ \\
\hline
\end{tabular}

Table 3 Carcinoma breast: correlation of stromal changes/ response after neoadjuvant therapy

\begin{tabular}{|l|l|}
\hline Stromal change/response & Number of cases (\%) \\
\hline Elastosis/collagenization & $14(35.9)$ \\
\hline Fibrosis & $25(64.1)$ \\
\hline Necrosis & $29(74.4)$ \\
\hline Desmoplasia & $23(59)$ \\
\hline $\begin{array}{l}\text { Hyalinization of the walls of blood } \\
\text { vessels }\end{array}$ & $2(5.1)$ \\
\hline Calcification & $9(23.1)$ \\
\hline Mucinous change & $3(7.7)$ \\
\hline Foamy histiocytes & $8(20.5)$ \\
\hline Cancerization of lobules & $10(25.6)$ \\
\hline Angiogenesis & $9(23.1)$ \\
\hline Giant cells & $14(35.9)$ \\
\hline $\begin{array}{l}\text { Atrophy of adjacent breast } \\
\text { parenchyma }\end{array}$ & $7(17.9)$ \\
\hline Degenerative changes & $13(33.3)$ \\
\hline Hemosiderin-laden macrophages & $7(17.9)$ \\
\hline
\end{tabular}

more commonly changes are described after neoadjuvant chemotherapy (also, termed primary systemic therapy or presurgical therapy) in which chemotherapy and hormonal and targeted therapies are administered before the actual surgical excision of the tumor. To have a better insight about the histopathologic changes induced by chemotherapy on a breast cancer, this study analyzed a series of breast cancer cases before and after the therapy. Clinical and histopathologic details were collected from a total of 39 cases of breast carcinoma before and post-therapy, and the changes induced by the therapy were correlated. Overall, breast cancer is the second most common cancer with India contributing to approximately $7 \%$ of the global burden. ${ }^{4,5}$ Because age is one of the most important risk factor for breast cancers, the incidence of breast cancers is seen to increase with age with most women getting diagnosed with breast cancers after the age of 40 years in their lives. In this study, the median age of breast carcinoma patients was found to be 55 years in contrast to the studies conducted by Sethi et $\mathrm{al}^{6}$ and Faneyte et $\mathrm{al}^{7}$ wherein the median age of breast cancer patients was found to be 46 and 45 years, respectively. In this study, the most common chief complaint was a palpable swelling in the affected breast whereas mastalgia (38.5\%), discharge from nipple, and ulcer were the other common complaints noted. The right breast was more commonly involved (66.7\%), and upper and outer quadrant was the most common location of the tumor (74.4\%) followed by upper and inner quadrant (8\%). In $13 \%$ of the cases, more than one quadrant was involved. This was in concordance with most of the reported studies wherein upper and outer quadrant was the most common location of the tumor. ${ }^{6,8}$ Interestingly, in this study, the mean size of the tumor before induction of chemotherapy was found to be $3.75 \mathrm{~cm}$ whereas after chemotherapy it was $1.75 \mathrm{~cm}$ $(p<0.001)$, indicating a reduction in mean size of the tumor by $50 \%$ seen in both small and large tumors in contrast with the study conducted by Fisher et $\mathrm{al}^{9}$ wherein 70 to $80 \%$ of the patients demonstrated $>50 \%$ decrease in the mean size of the tumor. As it is a known fact that IDC-NOC is the predominant histologic type seen in any breast cancer, IDC-NOC was the most common histologic type seen both before (94.87\%) and after (76.92\%) therapy in this study whereas one case each of lobular carcinoma and metaplastic carcinoma was also reported in conjunction with most of the previous studies. ${ }^{6,10-13}$ Therapy and prognosis in breast cancers largely depend on the stage at which the cancer is diagnosed. Breast cancer stage is one of the most important prognostic indicators and significant determinant of the patient's overall survival. Stage II breast carcinoma was found to be the most predominant (61.5\%) stage with stage IIA accounting for up to $38.5 \%$ of the cases, and $23 \%$ of the cases were found to be in stage III in this study. It was observed that younger women had an aggressive breast cancer with stage III as the predominant stage noted. These findings were in accordance with the numerous other studies conducted including the one conducted by Orucevic et al ${ }^{14}$ wherein stage II breast carcinoma was found to be the most predominant type. Similarly, stage II was the predominant stage reported in the studies conducted by Faneyte et $\mathrm{al}^{7}$ and von Minckwitz et al ${ }^{15}$ with $41 \%$ and $52 \%$ of the total cases presenting with stage II tumors, respectively. Narendra and Ray ${ }^{16}$ and Gajdos et al, ${ }^{17}$ however, reported most cases belonging to stage III in their studies in contrast to the aforementioned studies. Chemotherapy-induced morphologic changes were first described by Waller in 1960 when he described cytoplasmic swelling and vacuolation caused by administration of busulfan. Kennedy et al in 1990 further described such changes in patients with breast carcinoma when combination of tamoxifen and cytotoxic drug therapy was used. Since then, many studies have been published regarding the interaction of chemotherapeutic agents with tumor biology; however, there are very few studies that are detailed enough to include all the parameters such as necrobiotic changes, viability of the tumor cells and host tissue response to chemotherapeutics agents, etc., which could predict response to chemotherapy. In this study, an elaborative assessment of the cellular and stromal changes was done before and after the therapy. In the stromal changes, 
generalized increase in collagenization was significantly correlated with pathologic response and grades of tumor regression. The predominant stromal changes seen post-therapy included necrosis (74.4\%), fibrosis (64.1\%), desmoplasia (59\%), and degenerative changes (33.3\%) whereas mucinous changes, hyalinization of walls of blood vessels, neo-angiogenesis, calcific deposits, loss of cellular architecture, and appearance of chronic inflammatory cell infiltration and hemosiderinladen macrophages, giant cells, and histiocytes were the other significant stromal changes reported. Sethi et $\mathrm{al}^{6}$ reported generalized increase in mucinous and metaplastic changes left after chemotherapy. Honkoop et $\mathrm{al}^{12}$ also noticed similar mucinous changes left after chemotherapy. This was also in contrast to numerous other studies that did not report any significant histopathologic changes post-chemotherapy. ${ }^{12,18,19}$ Gazet et $\mathrm{al}^{20}$ also reported that lobular carcinoma was less responsive to chemotherapy due to high stromal content; however, the same could not be substantiated in this study because only one case each of lobular carcinoma and metaplastic carcinoma was reported in this study. The fundamental manifestation of the effect of therapy was an obvious decrease in tumor cellularity whereas the other prominent changes included nuclear enlargement, hyperchromasia, and increased $\mathrm{N}: \mathrm{C}$ ratio that were found in up to $85 \%$ of the cases followed by presence of prominent nucleoli and karyorrhexis/karyolysis noted in 70 to $75 \%$ of the cases. Similar changes have also been observed independently in numerous other studies. ${ }^{6}{ }^{14}$ Such cytologic changes are seen because of the cellular damage seen secondary to neoadjuvant chemotherapy as has been hypothesized in other studies and are variable in different tumors after the therapy, and the actual change might further differ on the chemotherapy regimen used., ${ }^{921,22}$ The true biologic significance of such cytopathic changes in residual breast cancers, however, has received little attention and is poorly understood. ${ }^{23,24}$ Various inflammatory infiltrates might also be seen due to host tissue response to the necrobiotic tumor such as appearance of chronic inflammatory cell infiltration and hemosiderin-laden macrophages, intense lymphocytic reactions, presence of plasma cells, and giant cells, and histiocytes as described in various studies. ${ }^{12,20,21,24,25}$ Stromal elastosis too is seen as a prominent change after therapy.,11,12,20,21 In this study, response to chemotherapy was classified according to the International Union against Cancer (UICC) as pCR defined as absence of residual viable tumor cells (DCIS included), pPR as greater than $50 \%$ reduction in the number of viable tumor cells, SD as less than $50 \%$ reduction in the number of viable tumor cells, or no change and progressive disease (PD) as an increase of at least $25 \%$ of viable tumor cells. On histopathologic examination, $\mathrm{pCR}$ was observed in $18 \%$ of the cases in this study whereas $15 \%$ showed pPR and $66.7 \%$ cases had an SD with no progression of disease after therapy. $\mathrm{pCR}$ was seen in seven of the cases of IDC-NOC type of the tumor whereas one case each of lobular, metaplastic, and ductal carcinoma had pPR. Twenty-six cases revealed pNR whereas response in IDCNOC type of tumor was not significantly different from that observed in invasive lobular carcinoma.

\section{Conclusion}

Breast cancer therapy causes morphologic alterations in the cancerous as well as the surrounding healthy tissue. The histopathologic interpretation in such cases, thus, requires a thorough knowledge of the cytologic and stromal changes rendered by the therapy. This ensures the role of the pathologists extremely important in correct diagnosis as well as grading of the tumor with a correct histopathologic interpretation for an effective and planned regimen of the therapy increasing prognosis and chances of survival of the affected patients rendering better clinical outcome and an effective patient care.

\section{Funding}

None.

\section{Conflict of Interest}

None declared.

\section{Acknowledgments}

The authors would like to thank all the patients who contributed in the study without whom this study would not have been feasible.

\section{References}

1 Babita R, Kumar N, Karwasra RK, Singh M, Malik JS, Kaur A Reproductive risk factors associated with breast carcinoma in a tertiary care hospital of north India: a case-control study. Indian J Cancer 2014;51(3):251-255

2 Zhang Q Ma B, Kang M. A retrospective comparative study of clinicopathological features between young and elderly women with breast cancer. Int J Clin Exp Med 2015;8(4):5869-5875

3 Leong AS, Zhuang Z. The changing role of pathology in breast cancer diagnosis and treatment. Pathobiology 2011;78(2):99-114

4 Doval DC, Sharma A, Sinha R, et al. Immunohistochemical profile of breast cancer patients at a tertiary care hospital in New Delhi, India. Asian Pac J Cancer Prev 2015;16(12):4959-4964

5 Gabriel CA, Domchek SM. Breast cancer in young women. Breast Cancer Res 2010;12(5):212

6 Sethi D, Sen R, Parshad S, Khetarpal S, Garg M, Sen J. Histopathologic changes following neoadjuvant chemotherapy in locally advanced breast cancer. Indian J Cancer 2013; 50(1):58-64

7 Faneyte IF, Schrama JG, Peterse JL, Remijnse PL, Rodenhuis S, van de Vijver MJ. Breast cancer response to neoadjuvant chemotherapy: predictive markers and relation with outcome. Br J Cancer 2003;88(3):406-412

8 Manjer J, Balldin G, Garne JP. Tumour location and axillary lymph node involvement in breast cancer: a series of 3472 cases from Sweden. Eur J Surg Oncol 2004;30(6):610-617

9 Fisher B, Brown A, Mamounas E, et al. Effect of preoperative chemotherapy on local-regional disease in women with operable breast cancer: findings from National Surgical Adjuvant Breast and Bowel Project B-18. J Clin Oncol 1997;15(7):2483-2493

10 Bonadonna G, Valagussa P, Zucali R, Salvadori B. Primary chemotherapy in surgically resectable breast cancer. CA Cancer J Clin 1995;45(4):227-243

11 Rasbridge SA, Gillett CE, Seymour AM, et al. The effects of chemotherapy on morphology, cellular proliferation, apoptosis and oncoprotein expression in primary breast carcinoma. Br J Cancer 1994;70(2):335-341 
12 Honkoop AH, Pinedo HM, De Jong JS, et al. Effects of chemotherapy on pathologic and biologic characteristics of locally advanced breast cancer. Am J Clin Pathol 1997;107(2):211-218

13 Burcombe RJ, Makris A, Richman PI, et al. Evaluation of ER, PgR, HER-2 and Ki-67 as predictors of response to neoadjuvant anthracycline chemotherapy for operable breast cancer. Br J Cancer 2005;92(1):147-155

14 Orucevic A, Chen J, McLoughlin JM, Heidel RE, Panella T, Bell $\mathrm{J}$. Is the TNM staging system for breast cancer still relevant in the era of biomarkers and emerging personalized medicine for breast cancer-an institution's 10-year experience. Breast J 2015;21(2):147-154

15 von Minckwitz G, Costa SD, Eiermann W, et al. Maximized reduction of primary breast tumor size using preoperative chemotherapy with doxorubicin and docetaxel. J Clin Oncol 1999;17(7):1999-2005

16 Narendra H, Ray S. Breast conserving surgery for breast cancer: single institutional experience from Southern India. Indian J Cancer 2011;48(4):415-422

17 Gajdos C, Tartter PI, Estabrook A, Gistrak MA, Jaffer S, Bleiweiss IJ. Relationship of clinical and pathologic response to neoadjuvant chemotherapy and outcome of locally advanced breast cancer. J Surg Oncol 2002;80(1):4-11

18 van der Wall E, Rutgers EJ, Holtkamp MJ, et al. Efficacy of up-front 5-fluorouracil-epidoxorubicin-cyclophosphamide (FEC) chemotherapy with an increased dose of epidoxorubicin in high-risk breast cancer patients. Br J Cancer 1996;73(9):1080-1085
19 Demaria S, Volm MD, Shapiro RL, et al. Development of tumor-infiltrating lymphocytes in breast cancer after neoadjuvant paclitaxel chemotherapy. Clin Cancer Res 2001;7(10):3025-3030

20 Gazet JC, Coombes RC, Ford HT, et al. Assessment of the effect of pretreatment with neoadjuvant therapy on primary breast cancer. Br J Cancer 1996;73(6):758-762

21 Morrow M, Braverman A, Thelmo W, et al. Multimodal therapy for locally advanced breast cancer. Arch Surg 1986;121(11):1291-1296

22 Niu S, Wen G, Ren Y, et al. Predictive value of primary tumor site for loco-regional recurrence in early breast cancer patients with one to three positive axillary lymphadenopathy. J Cancer 2017;8(12):2394-2400

23 Rajan R, Esteva FJ, Symmans WF. Pathologic changes in breast cancer following neoadjuvant chemotherapy: implications for the assessment of response. Clin Breast Cancer 2004;5(3):235-238

24 Moreno A, Escobedo A, Benito E, Serra JM, Gumà A, Riu F. Pathologic changes related to CMF primary chemotherapy in breast cancer. Pathological evaluation of response predicts clinical outcome. Breast Cancer Res Treat 2002;75(2):119-125

25 Moon YW, Rha SY, Jeung HC, Yang WI, Suh CO, Chung HC. Neoadjuvant chemotherapy with infusional 5-fluorouracil, Adriamycin and cyclophosphamide (iFAC) in locally advanced breast cancer: an early response predicts good prognosis. Ann Oncol 2005;16(11):1778-1785 